\title{
A Medicina de Família e Comunidade, a Atenção Primária à Saúde e o Ensino de Graduação: recomendações e potencialidades
}

\author{
Family and Community Medicine, \\ Primary Care and Undergraduate Medical \\ Education: recommendations and potentialities
}

Maria Inez Padula Anderson ${ }^{1}$

Marcelo Marcos Piva Demarzo ${ }^{2}$

Ricardo Donato Rodrigues ${ }^{3}$

\begin{abstract}
Resumo
Este documento tem por objetivo apresentar a importância e as potencialidades da Medicina de Família e Comunidade (MFC) e, conseqüentemente, da Atenção Primária à Saúde, no curso de graduação em Medicina, em especial no momento de reforma do ensino médico brasileiro, à luz das diretrizes curriculares nacionais para o curso de Medicina. Ele destaca os principais conteúdos teóricos e práticos atinentes à Medicina de Família e Comunidade recomendados para a graduação e as formas de organização institucional mais adequadas ao seu desenvolvimento e à sua implementação e, por último, relaciona os resultados mais relevantes das I e II Mostras Brasileiras de Medicina de Família e Comunidade e Graduação, realizadas em setembro de 2005 e 2006, como também uma síntese dos resultados da Oficina "Desafios do Ensino e da Aprendizagem da Atenção Primária à Saúde e da Medicina de Família e Comunidade na Graduação e Pós-Graduação em Medicina", realizada durante o Congresso da Associação Brasileira de Educação Médica de 2007. Pretende-se que este documento possa contribuir para a consolidação deste campo de saber e prática no âmbito da formação médica, como acontece na grande maioria das escolas médicas de todo o mundo, principalmente naquelas que implementaram reformas competentes no ensino e nos sistemas de saúde no sentido de os tornarem mais adequados às necessidades de saúde da população.
\end{abstract}

\begin{abstract}
The objective of this paper is to show the importance and potentialities of Family and Community Medicine (FCM) and consequently of Primary care in undergraduate medical education, particularly in a moment when the Brazilian medical courses are undergoing a reform in the light of the national course curriculum guidelines. The article highlights the principal theoretical and practical content related to Family and

Palavras-chave: Medicina de Família e

Comunidade; Atenção Primária à Saúde;

Educação Médica; Diretrizes.

Key Words: Family Practice; Primary Healthcare;

Medical Education; Medical Guidelines.

Médica de Família e Comunidade, Doutora em Saúde Coletiva, Professora adjunta e preceptora da Residência em MFC, FCM, UERJ, Presidente da SBMFC, Rio de Janeiro, Brasil.

${ }^{2}$ Médico de Família e Comunidade, Diretor de Graduação da SBMFC. Doutor em Ciências Médicas, Prof. Adj. Dep.de Medicina, UFSCAR, São Carlos, São Paulo, Brasil.

${ }^{3}$ Clínico Geral, Doutor em Saúde Coletiva, Professor adjunto e preceptor da Residência em MFC, FCM, UERJ, Presidente da AMFC-RJ, Rio de Janeiro, Brasil.
\end{abstract}


Community Medicine recommended for the undergraduate course and the forms of institutional organization most appropriate for its development and implementation. Finally, it refers to the most relevant results of the I and II Brazilian Exbibits on Family and Community Medicine and Undergraduate Medical Education, held in September 2005 and 2006, and of the Workshop "Challenges in Learning/ Teaching of Primary Care and Family and Community Medicine in the Undergraduate and Graduate Medical Education” beld during the Congress of the Brazilian Association of Medical Education in 2007. This article aims to contribute to the consolidation of this field of knowledge and practice in medical education as happens in most medial schools worldwide, mainly where medical education and health systems underwent competent reforms for meeting the bealth needs of the population.

\section{Introdução}

A Medicina de Família e Comunidade (MFC) é uma especialidade eminentemente clínica que também desenvolve, de forma integrada e integradora, práticas de promoção, proteção e recuperação da saúde, dirigidas a pessoas, famílias e comunidades. Esses atributos a tornam uma disciplina estratégica para a ressignificação das bases estruturais da própria profissão médica, adquirindo papel fundamental na constituição dos novos paradigmas em Saúde.

A MFC tem potencial transformador tanto no âmbito da prática médica quanto na formação de recursos humanos e no desenvolvimento de pesquisas, contribuindo para uma maior efetividade dessas áreas, inspiradas em bases mais humanas e comunitárias. Ela tem, também, assumido papel relevante principalmente na promoção de Atenção Integral à Saúde.

Seus preceitos, conceitos e direcionamentos relacionam-se aos da Atenção Primária à Saúde (APS), de tal modo que é reconhecida internacionalmente como a especialidade médica de excelência da APS, sendo estratégica para o pleno desenvolvimento da mesma. Por isso, tem sido considerada, assim como a APS, uma especialidade médica com potencial estruturante para os Sistemas Nacionais de Saúde, em especial, em cenário de crise e reforma sanitária, como o que vivencia- mos em nível nacional e internacional.

Os princípios e as práticas da MFC são centrados na "pessoa" (e não na "doença"), na relação entre médico e indivíduo, e na relação deste sujeito, mais ou menos sadio, com sua família e com a comunidade em que vive. A MFC aborda o processo saúde-adoecimento como um fenômeno complexo, relacionado à interação de fatores biológicos, psicológicos, socioambientais e espirituais, sendo, portanto, um processo influenciado fortemente pela estrutura familiar e comunitária do indivíduo.

\section{Princípios da Medicina de Família e Comunidade}

Com base em princípios, conceitos e recomendações internacionais formalizados pela Organização Mundial dos Médicos de Família - WONCA ${ }^{1}$-, a especialidade Medicina de Família e Comunidade tem por objetivos:

- Atuar, prioritariamente, no âmbito da Atenção Primária à Saúde (APS), a partir de uma abordagem biopsicossocial e existencial do processo saúde-adoecimento.

- Desenvolver ações integradas de promoção, proteção, recuperação da saúde, no nível individual e coletivo.

- Priorizar a prática médica centrada na pessoa, na relação médico-paciente, com foco na família e orientada para comunidade, privilegiando o acesso, o primeiro contato, o vínculo, a continuidade e a integralidade do cuidado na atenção à saúde. - Coordenar os cuidados de saúde prestados a determinado indivíduo, família e comunidade, referenciando, sempre que necessário, para outros especialistas ou outros níveis e setores do sistema, mas sem perda do vínculo.

- Atender, com elevado grau de qualidade e resolutividade, no âmbito da Atenção Primária à Saúde, cerca de $85 \%$ dos problemas de saúde relativos a uma população específica, sem diferenciação de gênero ou faixa etária.

- Desenvolver, planejar, executar e avaliar, junto à equipe de saúde, programas integrais de atenção, objetivando dar respostas adequadas às necessidades de saúde de uma população adscrita, tendo por base metodologias apropriadas de investigação, com ênfase na utilização do método científico e epidemiológico.

- Estimular a resiliência, a participação e a autonomia dos 
indivíduos, das famílias e da comunidade.

- Desenvolver novas tecnologias em Atenção Primária à Saúde.

- Desenvolver habilidades no campo da metodologia pedagógica e a capacidade de auto-aprendizagem e empoderamento dos indivíduos.

- Desenvolver a capacidade de atuação médica humanizada, relevando seus aspectos científicos, éticos e sociais.

\section{A MFC e a Graduação em Medicina}

\section{Recomendações internacionais}

Segundo Byrne e colaboradores², há sete razões principais para incluir a Medicina de Família e Comunidade (MFC) nas universidades, particularmente no Ensino de Graduação em Medicina:

1. Os estudantes de Medicina devem vivenciar e entender o cenário e as maneiras pelas quais a grande maioria da população é cuidada pelos serviços de saúde.

2. Os estudantes de Medicina devem vivenciá-la na sua forma mais integrada e integradora, o que é mais bem evidenciado no cenário de prática do Médico de Família e Comunidade.

3. Os estudantes de Medicina devem vivenciá-la centrada na pessoa (um dos pilares da Medicina de Família e Comunidade), invertendo a tendência de enfoque na doença e na tecnologia dura.

4. Os estudantes de Medicina devem ter a chance de vivenciar a prática da MFC, a fim de poderem incluir essa especialidade na lista de opções para a futura carreira profissional. Os estudantes de Medicina que têm perfil e podem se tornar futuros médicos de família e comunidade necessitam ter contato com esta especialidade, a exemplo do que ocorre em relação as outras especialidades no curso de graduação.

5. Estudantes de Medicina que seguirão outras especialidades, que não a MFC, necessitam conhecer as bases de atuação desta especialidade. Isso é particularmente importante se considerarmos que a maioria das especialidades atuará no sistema de saúde como referência para o encaminhamento e/ou parecer de casos provenientes dos médicos de família e comunidade, e/ou utilizarão os médicos de família e comunidade como referência para o acompanhamento clínico geral dos pacientes atendidos por eles.

6. A participação de médicos de família e comunidade como professores no ensino médico de graduação abre novas possibilidades de ensino, pesquisa e extensão à escola médica, ampliando o envolvimento e a responsabilidade da mesma com as necessidades e demandas de saúde das pessoas e das comunidades.

7. A MFC, como também as outras especialidades da profissão médica, necessita da vivência no Ensino de Graduação, possibilitando assim espaço e estímulo para o seu desenvolvimento acadêmico no sentido mais amplo.

O potencial transformador da Medicina de Família e Comunidade ("Medicina Integral, Geral, Familiar e/ou Comunitária” em outros países) para a graduação, principalmente, em Medicina tem sido evidenciado por organismos internacionais como a Organização Mundial de Saúde $(\mathrm{OMS})^{3}$ e a Associação Mundial dos Médicos Gerais e de Família (WONCA).

Isso pode ser comprovado pela inserção dos conceitos e das práticas da MFC na estrutura de destacadas escolas médicas de todo o mundo. Observa-se também que a grande maioria destas escolas constituiu Departamentos de Medicina de Família e Comunidade (ou denominações equivalentes), muitas com incentivo financeiro governamental, como no caso das escolas norte-americanas, o que ocorreu ainda na década de 70 do último século ${ }^{4}$. ${\text { A } \mathrm{OMS}^{1}}^{1}$ e a WONCA ${ }^{3}$ entendem que os fundamentos conceituais e éticos, as técnicas e práticas da Medicina de Família e Comunidade constituem elementos importantes na formação médica geral, independentemente da especialidade que o futuro médico irá exercer, envolvendo os estudantes em uma prespectiva ampliada do cuidado em saúde.

Os conteúdos programáticos ${ }^{1,3}$ dessa especialidade, recomendados para a graduação médica, e, recentemente, revisados pela Sociedade Norte-Americana de Professores de Medicina de Família (Society of Teachers of Family Medicine) $)^{5}$, segundo as diretrizes do projeto "Futuro da Medicina de Família" (Future of Family Medicine ${ }^{6}$ ), devem 
incluir:

- Conceituação e reconhecimento da importância da Atenção

Primária à Saúde, da Medicina Ambulatorial e da Medicina de Família e Comunidade nos Sistemas Nacionais de Saúde.

- Compreensão e incorporação dos princípios e das atitudes envolvidas com a complementaridade e integralidade das ações médicas e de saúde.

- Atuação em cenários nos quais sejam desenvolvidas práticas de Atenção Primária à Saúde (como por exemplo, a Estratégia de Saúde da Família).

- Reconhecimento do papel e da influência da família no estado de saúde de seus componentes.

- Comunicação e relação Médico-Paciente-FamíliaComunidade.

- Reconhecimento e elaboração do diagnóstico de saúde biopsicossocial da família e da comunidade.

- Conhecimento do cadastro familiar e comunitário como instrumento facilitador do diagnóstico e da abordagem familiar e comunitária.

- Conhecimento e desenvolvimento de práticas de promoção, proteção e educação em saúde da população, com desenvolvimento de ações de saúde orientadas pelas necessidades e demandas percebidas por meio do contato com as famílias e a comunidade.

- Estudo e reflexão sobre os problemas de saúde que mais afetam as pessoas e as populações de centros urbanos e rurais. - Práticas e metodologias próprias da clínica da medicina ambulatorial.

- Cuidado humanizado e efetivo aos pacientes e suas famílias para a resolução de problemas de saúde e para a promoção de saúde.

- Prática voltada ao sistema de saúde nacional, com compromisso ético e profissional com a saúde da população do próprio país, racionalizando recursos e ações e melhorando os indicadores de saúde populacionais.

Assim, as competências a serem desenvolvidas nos estudantes são:

- Convivência e colaboração com profissionais de outras profissões e especialidades para prover cuidado centrado na pessoa e nos aspectos preventivos, durante todas as fases da vida.

- Sensibilidade e responsabilidade em relação ao paciente, a sua cultura, idade, gênero e disabilidades.

- Prática de diagnóstico e terapêutica com base em evidência científica e com participação do paciente e de sua família, informando-os apropriadamente e envolvendo-os no processo.

- Acolhimento e resolutividade aos agravos agudos e crônicos mais comuns, segundo protocolos clínicos embasados cientificamente, e envolvendo os principais grupos de cuidado (crianças e adolescentes, mulheres, adultos e idosos).

- Manejo dos agravos de saúde numa abordagem multiprofissional e interdisciplinar, envolvendo também a família, e tendo como objetivo a melhora funcional e de qualidade de vida da pessoa.

- Prática da educação em saúde dos pacientes, suas famílias e comunidade, aconselhando sobre os hábitos de vida inadequados à saúde e aplicando estratégias adequadas de abordagem do problema, visando a mudanças de comportamentos e considerando a cultura de cada pessoa e população.

- Descrição da prevalência e a história natural dos problemas de saúde mais comuns, tanto ao nível individual quanto familiar e comunitário.

- Reflexão e discussão sobre o cuidado integral, contínuo e integrado para as pessoas, além das dificuldades inerentes a esse processo.

- Identificação dos vários níveis de prevenção e aplicação das ações preventivas em cada nível, segundo embasamento científico.

- Diferenciação das características epidemiológicas dos vários níveis de atenção.

- Reconhecimento e interpretação das leis públicas mais relevantes referentes à promoção de saúde das populações. - Entendimento da complexidade do diagnóstico feito muitas vezes frente à escassez de dados clínicos.

- Manejo adequado da tecnologia de informação em saúde.

- Treinamento de técnicas de comunicação adequadas para trabalho em equipe multiprofissional e para a educação e informação em saúde dos pacientes e familiares.

- Reconhecimento dos limites do conhecimento pessoal, 
porém, sempre coordenando e advogando pelo cuidado adequado à população dentro do sistema de saúde nacional.

- Reconhecimento das barreiras físicas, culturais e administrativas para o cuidado integral e adequado às pessoas.

Em 2002, a WONCA Europa publicou a nova Definição Européia de Medicina Familiar ${ }^{1}$. As implicações deste trabalho para a formação e investigação em Medicina Familiar foram profundas. A EURACT (Academia Européia de Professores de Medicina Familiar) continuou a trabalhar nas questões educativas que surgiram e, como primeiro passo, elaborou uma «agenda educativa» ${ }^{7}$. A designação de «agenda» foi deliberada. O termo significa que ela constitui simultaneamente uma revisão dinâmica do ensino e aprendizagem da Medicina Familiar e uma identificação do trabalho que tem ainda de ser feito: o outro elemento da "agenda".

Segundo a Agenda Educativa da EURACT (AEE), a disciplina de Medicina de Família e Comunidade precisa definir:

- Como se aprende melhor a MFC?

- Onde deve ser ensinada?

- Quando deve ser ensinada?

- O que deve ser ensinado?

- O que deve a MFC ensinar aos formandos de outras disciplinas?

Segundo a $\mathrm{AEE}^{7}$, a $\mathrm{MFC}$ tem uma contribuição a dar ao ensino de todos os médicos em todas as fases da sua formação. Geralmente, ocorrem mudanças de atitude nos alunos de Medicina depois de terem tido oportunidade de resolver por si problemas de cuidados primários e observar como os seus professores resolvem os problemas que se apresentam e eles. O contato precoce dos alunos de Medicina com a clínica por períodos breves de alguns dias a várias semanas, no início do curso de Medicina, pode lançar as bases que permitem ao estudante de Medicina apreender o sentido de toda a formação médica, aprendendo sobre as pessoas no contexto dos seus problemas de saúde. Aulas ou pequenos seminários no período pré-clínico podem ser eficazes para dar ao aluno determinados conhecimentos necessários antes de começar a trabalhar com pacientes e suas famílias.

A definição da disciplina de MFC deve conduzir diretamente às competências nucleares do médico de família. Nucleares significa essenciais à disciplina, independentemente do sistema de saúde em que as competências são aplicadas ${ }^{7}$. As 11 características da disciplina referem-se às 11 aptidões que todos os especialistas de MFC devem dominar. Dadas as suas inter-relações, agrupam-se em seis categorias independentes de competências nucleares (Figura 01).

\section{Gestão em Cuidados Primários \\ Inclui a capacidade para:}

- gerir o contato primário com os pacientes, lidando com problemas não-selecionados;

- cobrir todo o leque de problemas de saúde;

- coordenar os cuidados com outros profissionais dos cuidados primários e com outros especialistas;

- dominar a prestação eficaz e adequada de cuidados de saúde e a utilização dos serviços de saúde;

- disponibilizar ao paciente os serviços adequados dentro do sistema de saúde;

- atuar como advogado do paciente.

\section{Cuidados centrados na pessoa}

Inclui a capacidade para:

- adotar uma abordagem centrada na pessoa ao lidar com os pacientes e os seus problemas no contexto das circunstâncias do paciente;

- desenvolver e aplicar a consulta de clínica geral para promover uma eficaz relação médico-paciente, com respeito pela autonomia do paciente;

- comunicar, estabelecer prioridades e atuar em parceria; - proporcionar continuidade longitudinal de cuidados tal como a determinarem as necessidades do paciente no que se refere à gestão continuada e coordenada de cuidados.

3. Aptidões para a resolução de problemas específicos Inclui a capacidade para:

- relacionar os processos específicos de decisão com a prevalência e incidência das doenças na comunidade; 
- reunir e interpretar seletivamente a informação recolhida na anamnese, exame objetivo e exames complementares e aplicá-la a um plano de ação adequado em colaboração com o paciente;

- adotar princípios de trabalho adequados, por exemplo, pedindo exames complementares de modo seqüencial, e usando o tempo como um instrumento e como modo de tolerar a incerteza;

- intervir com urgência quando necessário;

- gerir as situações que se apresentam precocemente e de forma indiferenciada;

- utilizar as intervenções diagnósticas e terapêuticas de modo efetivo e eficiente.

4. Abordagem abrangente

Inclui a capacidade para:

- gerir simultaneamente múltiplas queixas e patologias, e tanto problemas de saúde agudos como crônicos do indivíduo;

- promover a saúde e o bem-estar aplicando adequadamente as estratégias de promoção da saúde e prevenção da doença; - gerir e coordenar a promoção da saúde, prevenção, cura, tratamento, paliação e reabilitação.

\section{Orientação Comunitária}

Inclui a capacidade para:

- conciliar as necessidades de cada paciente e as necessidades de saúde da comunidade em que ele vive, de acordo com os recursos disponíveis.

\section{Abordagem holística}

Inclui a capacidade para:

- usar um modelo biopsicossocial levando em conta as dimensões cultural e existencial.
Figura 01 - Adaptada de "WONCA tree" College of Primary Care Medicine/U. Grueninger. Referido na agenda da EURACT como a "árvore da sabedoria":

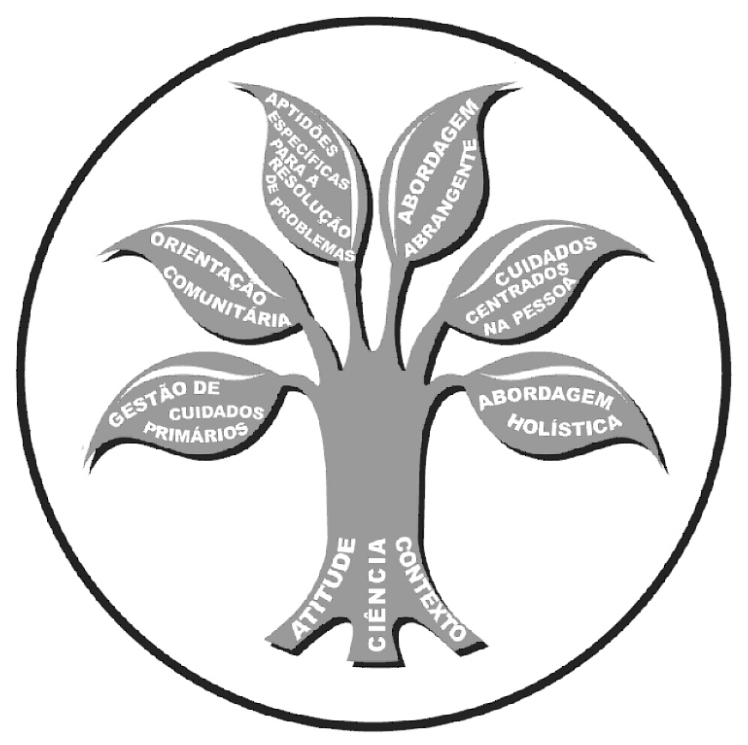

Fonte: www.kollegium.ch (C) 2004

\section{Os Departamentos de Medicina de Família e Co- munidade}

Segundo orientação da Organização Mundial da Saúde em conjunto com a Associação Mundial de Médicos de Familia ${ }^{5}$, implantar Departamentos ou Unidades ou Atividades Curriculares de MFC permite o direcionamento necessário para a inclusão da disciplina no espaço acadêmico, como também permite a organização dos recursos que são necessários para articularem a tríade ensino, cuidado ao paciente (extensão) e programas de pós-graduação e pesquisa.

Os tópicos a seguir fazem parte da publicação acima relacionada e pretendem contribuir para a criação de estruturas departamentais ou unidades docentes de MFC.

Segundo estas instituições, estabelecer Departamentos de Medicina de Família requer a participação de lideranças que compreendam e apóiem as importantes funções e papéis da Medicina de Família. Essas lideranças incluem autoridades governamentais, associações médicas, médicos de família, staffs das escolas médicas e dos hospitais universitários, além de profissionais da saúde pública.

Recomendam também que, desde o início, é impor- 
tante desenvolver um bom relacionamento com outros departamentos existentes, como os de Medicina Interna e Pediatria, que, compartilhando interesses, podem implementar um processo de colaboração mútua visando à implementação de atividades de ensino e de projetos de pesquisa interdisciplinares. Estes especialistas freqüentemente colaboram para o aprendizado do médico de família, e muitos têm consciência que são mais efetivos quando trabalham articulados com médicos de família capacitados. As Escolas Médicas parecem mais propensas ao desenvolvimento da Medicina de Família quando esta disciplina é vista como essencial para potencializar a função dos outros profissionais.

Ainda segundo as orientações da OMS e WONCA, a constituição de Departamentos de Medicina de Família requer um provimento adequado de recursos humanos e físicos, de modo a desenvolver o amplo espectro dos programas de educação, assistência e pesquisa da MFC. Os recursos físicos devem incluir centros que integrem as atividades assistenciais e de ensino - nestes centros, o cuidado ao paciente deve ser provido por intermédio de equipes que incluem médicos de MFC, residentes e/ou estudantes em treinamento, enfermeiras, assistentes sociais e outros profissionais de saúde. Os Centros de ensino de Medicina de Família podem e devem ser incorporados às unidades assistenciais do sistema de saúde (integração ensino-serviço-comunidade) e podem também servir como importante local de desenvolvimento de pesquisa na Atenção Primária.

Os Departamentos de Medicina de Família freqüentemente requerem apoio governamental e institucional, inclusive de ordem financeira. Usualmente, estes departamentos iniciam com pequeno número de especialistas e profissionais, crescendo à medida do desenvolvimento dos programas clínicos e de pesquisa. Os hospitais universitários e outros são importantes parceiros para o desenvolvimento e suporte destes departamentos de Medicina de Família, ao mesmo tempo em que estes os especialistas em MFC e os programas de residência prestam relevantes serviços para estas instituições.

A Medicina de Família e Comunidade e as diretrizes curriculares nacionais do curso de graduação em Me- dicina.

À semelhança do que acontece em muitos países desenvolvidos, a inserção da MFC nos currículos de graduação já constitui uma realidade em um número considerável de escolas médicas brasileiras. Entretanto, ainda é necessário consolidar, adequar, qualificar e expandir este processo.

Este movimento de inserção se tornou mais relevante, a partir das Diretrizes Curriculares Nacionais do Curso de Graduação em Medicina $(\mathrm{DCN})^{8}$, desde quando vêm ocorrendo mudanças mais consistentes no ensino médico em nosso país. A Medicina de Família e Comunidade tem tido importante papel nesse processo, tendo em vista a superposição entre seus princípios e práticas e as recomendações incorporadas às Diretrizes, a exemplo do que consigna seu artigo $3^{\circ}$ :

Art. $3^{\circ} \mathrm{O}$ Curso de Graduação em Medicina tem como perfil do formando egresso/profissional o médico, com formação generalista, humanista, crítica e reflexiva, capacitado a atuar pautado em princípios éticos, no processo de saúde-doença em seus diferentes níveis de atenção, com ações de promoção, prevenção, recuperação e reabilitação à saúde, na perspectiva da integralidade da assistência, com senso de responsabilidade social e compromisso com a cidadania, como promotor da saúde integral do ser humano.

Parágrafo Único. Com base nestas competências, a formação do médico deverá contemplar o sistema de saúde vigente no país, a atenção integral da saúde num sistema regionalizado e hierarquizado de referência e contrareferência e o trabalho em equipe.

Ou ainda:

Art. $6^{\circ}$ Os conteúdos essenciais para o Curso de Graduação em Medicina devem estar relacionados com todo o processo saúde-doença do cidadão, da família e da comunidade, integrado à realidade epidemiológica e profissional, proporcionando a integralidade das ações do cuidar em Medicina.

Conforme documento divulgado recentemente pelo Ministério da Educação em parceria com o Ministé- 
rio da Saúde (MEC/MS) ${ }^{9}$, os cursos de Medicina com maior grau de aderência aos princípios das DCN, além de outras características, apresentam a Estratégia Saúde da Família como uma das estratégias para a formação de um médico capaz de responder às necessidades sociais da população brasileira.

Apesar dos conceitos e práticas da MFC se apresentarem como relevante estratégia para a operacionalização das Diretrizes Curriculares Nacionais do Curso de Graduação em Medicina, ainda são necessários esforços governamentais para incentivar o desenvolvimento da MFC e da APS, no âmago das universidades. Além disso, e com a participação da SBMFC, as diretrizes para o conteúdo programático dessa inserção devem ser sistematizadas, segundo a realidade da saúde no Brasil.

Dentro dessa lógica, a SBMFC tem promovido encontros de sensibilização e trocas de experiências em nível nacional, abordando a questão da inserção acadêmica da MFC, e envolvendo vários protagonistas do processo, tais como professores, preceptores, residentes, estudantes, gestores e profissionais de saúde, e a comunidade. A seguir, são apresentados os resultados gerais dos eventos promovidos até então.

\section{I Mostra de Medicina de Família e Comunidade e Graduação}

Em setembro de 2005, durante o I Congresso Paulista de Medicina de Família e Comunidade, ocorreu a I Mostra Brasileira de Medicina de Família e Comunidade e Graduação, que teve como objetivo oferecer um espaço de discussão e troca de experiências entre as Instituições de Ensino Superior com interesse e práticas na atividade.

Também fez parte da I Mostra uma pesquisa, que visou a levantar dados sobre a situação atual da inserção da MFC na graduação médica brasileira. Os dados estão apresentados na forma de tabelas e quadros (abaixo). Instituições que enviaram experiências em MFC e Graduação:

- Curso de Medicina - Universidade Severino Sombra (Vassouras, RJ)

- Curso de Medicina - Centro Universitário Barão de Mauá
(Ribeirão Preto, SP)

- Faculdade de Ciências Médicas - Universidade Estadual do Rio de Janeiro

- Faculdade de Medicina - Universidade de Ribeirão Preto

- Faculdade de Medicina - Universidade de Uberaba (UNIUBE)

- Faculdade de Medicina - Universidade de São Paulo Campus Ribeirão Preto

- Faculdade de Medicina - Universidade de São Paulo Campus Capital

- Faculdade de Medicina - Universidade Souza Marques (Rio de Janeiro)

- Faculdade de Medicina de Marília (FAMEMA)

\section{Discussões e Conclusões da I Mostra}

Além da pesquisa e das experiências apresentadas durante a Mostra, também houve espaço para discussões e debates dentro do contexto da inserção atual da MFC na graduação em Medicina no Brasil. Apesar das dificuldades inerentes à organização e implementação sistematizada de um campo de conhecimento e prática na estrutura das escolas médicas, a opinião dos participantes foi unânime no sentido da importância desta inserção e da necessidade de desenvolvimento e consolidação desta estratégia.

Os principais eixos e pontos abordados foram:

1) Por que inserir os conceitos e práticas da MFC na graduação em Medicina?

- Estratégia para sensibilizar docentes e discentes em relação à especialidade MFC, fundamental para a reorganização do sistema de saúde público e suplementar no mundo e no Brasil.

- Estratégia para a formação de um médico mais humano e responsável em relação às pessoas, além de voltado à realidade do país.

- Embasar os estudantes para uma possível pós-graduação em MFC.

2) Como e em que momento inserir a MFC dentro da graduação?

- Criação de Departamentos de MFC.

- Inserção precoce e com enfoque prático. 
Tabela 1. Resultados gerais da pesquisa.

\author{
Número de Instituições Respondentes \\ Instituições Públicas de Ensino \\ Instituições Privadas de Ensino \\ Instituições com prática de MFC na Graduação \\ Tempo Médio da Experiência \\ Instituições com Departamento de MFC \\ Exemplos de outros Departamentos de Referência, \\ nos casos em que não havia um Departamento de MFC \\ Avaliação da Experiência pelas Instituições
}

Receptividade pelos Estudantes

Docentes com formação em MFC

(Residência Médica ou Especialização)

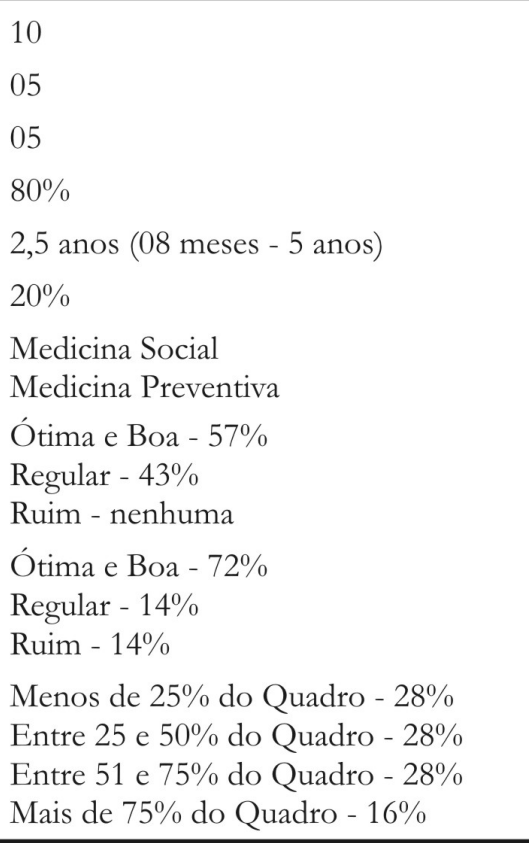

Quadro 1. Principais dificuldades apontadas pelas instituições respondentes quanto à experiência de inserção da MFC na graduação.

\footnotetext{
1. Docentes formados no Modelo Flexneriano.

2. Articulação incipiente com outras disciplinas do curso.

3. Dificuldade dos docentes no trabalho intersetorial.

4. Desconhecimento do corpo docente e discente em relação aos princípios da MFC (duas citações).

5. Preconceito / discordância / desvalorização do corpo discente e docente em relação à inserção da MFC na graduação

(05 citações), principalmente na experiência de inserção precoce no currículo.

6. Falta de recursos humanos com qualificação na área (duas citações).

7. Pequeno apoio logístico da Instituição de Ensino.

8. Dificuldade na relação ensino-serviço de saúde - Secretaria Municipal de Saúde, limitando a inserção do estudante nas

Unidades da Saúde Públicas (quatro citações).

9. Estudantes apenas observadores das atividades.

10. Tempo curto de estágio.
}

3) Quais seriam os maiores entraves?

- Relação conflituosa das instituições com o sistema de saúde local.

- Mesmos problemas apontados na pesquisa (quadro 01).

4) Como avaliar os resultados, quais indicadores?

- Avaliação e métodos ainda não totalmente sistematizados em relação à maioria das escolas, mas bastante desenvolvidos em algumas instituições.

As instituições participantes apresentaram muitos pontos convergentes, principalmente em relação às dificul- dades enfrentadas e às atividades desenvolvidas. Apesar do número de instituições na I Mostra não ser expressivo em termos nacionais, os resultados possivelmente refletem a realidade da inserção da MFC na graduação em Medicina no Brasil. Os resultados podem servir de base para discussões ampliadas junto às instituições de ensino, à Associação Brasileira de Educação Médica, ao MEC e ao Ministério da Saúde, na tentativa de gerar diretrizes e sistematizações sobre o tema, relevante para o futuro da Medicina no Brasil, como também para a consolidação do Sistema Único 
de Saúde Brasileiro, haja vista o incentivo à Estratégia Saúde da Família e as dificuldades para a formação de recursos humanos nessa nova lógica do sistema de saúde nacional.

\section{II Mostra de Medicina de Família e Comunidade} na graduação e I Encontro de Ligas Acadêmicas de MFC

Em setembro de 2006, durante o $44^{\circ}$ Congresso Brasileiro de Educação Médica ocorreu a II Mostra Brasileira de Medicina de Família e Comunidade e Graduação e o I Encontro de Ligas Acadêmicas de MFC e Saúde da Família, que tiveram como objetivo geral ampliar a discussão da inserção da MFC, dando prosseguimento aos trabalhos iniciados com a I Mostra de MFC na graduação ${ }^{12,13}$.

Os objetivos específicos foram:

1. Traçar um breve panorama da inserção atual da MFC e da APS nas escolas médicas brasileiras.

2. Reunir e discutir experiências nacionais e internacionais no tema.

3. Promover discussão coletiva (docentes, discentes e profissionais de saúde) de recomendações para a inserção de competências da MFC nos currículos de graduação em Medicina.

4. Discutir a inserção da MFC como área essencial no ensino médico de graduação em sintonia com as proposições das Diretrizes Curriculares Nacionais, considerando as convergências das competências relativas à MFC com as referidas diretrizes.

Houve diversos tipos de atividades:

1) Exposição de experiências acadêmicas, na forma de pôsteres, sobre a inserção da MFC na Graduação, segundo a vivência das diferentes instituições de ensino representadas.

2) Apresentação dos resultados do questionário, respondidos on-line pelos participantes no momento da inscrição, sobre o estado atual da inserção da MFC na Graduação em Medicina.

3) Realização do I Encontro de Ligas Acadêmicas de MFC, que reuniu docentes e discentes interessados em desenvolver atividades extracurriculares relacionadas à MFC nas Faculdades de Medicina.

4) Grupos de discussão relativos às seguintes áreas temáticas:

a) Competências (atitudes, conhecimentos e habilidades) da MFC na Graduação em Medicina. Por quê?

b) Competências (atitudes, conhecimentos e habilidades) da MFC na Graduação em Medicina. Quais e como?

c) Competências (atitudes, conhecimentos e habilidades) da MFC na Graduação em Medicina e os currículos com metodologias ativas de ensino-aprendizagem. Como fazer?

d) Estruturação dos cenários de prática. Quais e como?

5) Apresentação de experiências internacionais sobre a inserção da MFC na Graduação em Medicina.

6) Mesa-redonda com a temática A MFC e as Diretrizes Curriculares Nacionais do Curso de Graduação em Medicina: caminhos e potencialidades.

7) Reunião para discussão da criação da Academia Brasileira de Educadores de Medicina de Família e Comunidade, proposta com base em associações similares na Europa e América do Norte.

Principais resultados e encaminhamentos:

1) Panorama geral sobre o estado atual da inserção da MFC na Graduação em Medicina, por meio da compilação das informações colhidas pelos questionários respondidos pelos participantes (tabela e quadro abaixo).

2) Participação efetiva de mais de 200 pessoas, entre docentes, discentes e profissionais de saúde, e mais de 70 instituições de ensino superior estiveram representadas.

3) Durante o I Encontro de Ligas, houve a criação da Associação de Ligas Acadêmicas de Saúde da Família e Medicina de Família e Comunidade, com a aprovação de seus estatutos, tendo sido eleita sua primeira Diretoria.

4) Aprovação da SBMFC como entidade brasileira responsável pela Tradução Oficial da Agenda EURACT para o português.

5) Realização da primeira reunião da Academia Brasileira de Educadores de Medicina de Família e Comunidade e dis- 
cussão inicial de seus estatutos.

6) Produção de edição especial da Revista Brasileira de Medicina de Família e Comunidade (RBMFC) sobre a II Mostra, reunindo os resumos e relatos completos de experiênci- as de inserção da MFC na graduação em Medicina, além de um artigo do representante da EURACT na II Mostra, e da Tradução Oficial da Agenda Educativa EURACT.

Tabela 02. Dados Gerais dos Questionários Respondidos pelos Docentes durante a Inscrição para a II Mostra de MFC na Graduação*.

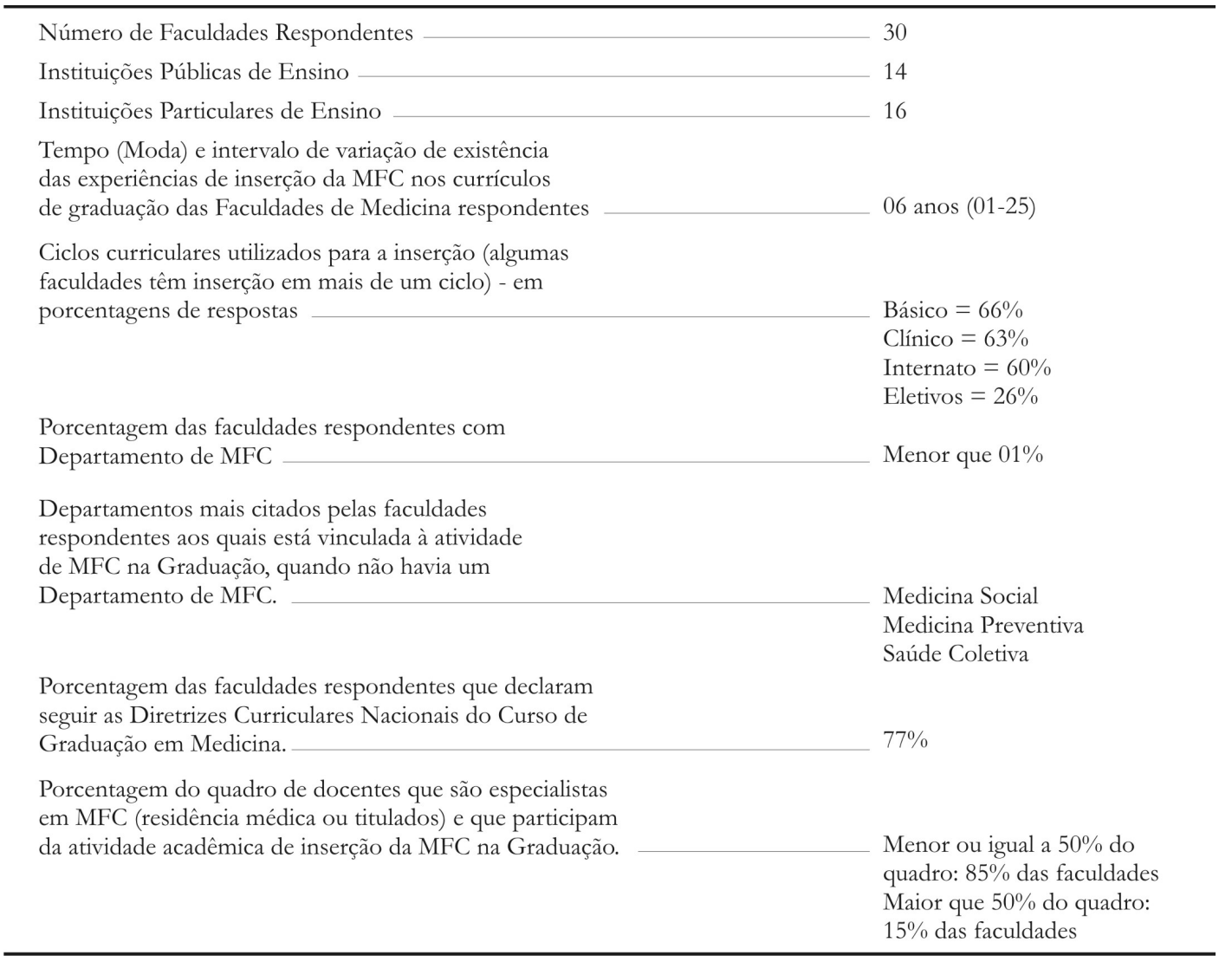

*Considerou-se apenas um questionário por Faculdade.

Quadro 02. Principais dificuldades apontadas pelos docentes respondentes quanto à experiência de inserção da MFC na Graduação*.

Organização inadequada da rede municipal de saúde $(03)^{* *}$

Docentes com capacitação inadequada para a especialidade MFC (06)

Número insuficiente de docentes envolvidos na atividade (05)

Resistência por parte dos discentes e pelos docentes de outras especialidades, resultando em apoio institucional insuficiente (08)

Infra-estrutura inadequada das unidades de saúde (11)

Desconhecimento da especialidade de MFC - Confusão entre MFC e Estratégia Saúde da Família (06)

Qualificação inadequada das equipes de saúde (04)

Pouco entendimento das Diretrizes Curriculares Nacionais para o Curso de Graduação em Medicina (04)

Violência nas comunidades prejudicando a segurança e o acesso dos estudantes (02)

*Foram consideradas as idéias centrais das respostas, com validação por avaliador externo

* Freqüencia de aparecimento da idéia, quando maior que uma vez. 
8. I Oficina "Desafios do Ensino e da Aprendizagem da Atenção Primária à Saúde e da Medicina de Família e Comunidade na Graduação e Pós-Graduação em Medicina"

Em outubro de 2007, durante o $45^{\circ}$ Congresso Brasileiro de Educação Médica, ocorreu a I Oficina "Desafios do Ensino e da Aprendizagem da Atenção Primária à Saúde e da Medicina de Família e Comunidade na Graduação e Pós-Graduação em Medicina", que reuniu professores, preceptores, residentes, estudantes, profissionais e gestores da área da saúde em um evento preparatório para o "I Fórum de Formação em Medicina de Família e Comunidade e Atenção Primária à Saúde", em Fortaleza (CE) ${ }^{14}$.

Tendo-se em vista a opção brasileira (acertada) por um modelo de atenção à saúde fundamentado na Atenção Primária (APS) e a implantação das Diretrizes Curriculares Nacionais do Curso de Medicina (2001) que abriram fortes perspectivas para a efetivação da participação da Medicina de Família e Comunidade e da APS nos currículos e programas de graduação e pós-graduação no Brasil; e que essa inserção ainda é incipiente e carente de sistematização e incentivo, a Sociedade Brasileira de Medicina de Família e Comunidade (SBMFC), com o apoio da Associação Brasileira de Educação Médica (ABEM), idealizou a Oficina "Desafios do Ensino e da Aprendizagem da Atenção Primária à Saúde e da Medicina de Família e Comunidade na Graduação e PósGraduação em Medicina".

Assim, os objetivos principais da Oficina foram:

- Identificar desafios no processo de ensino e aprendizagem da APS e da MFC, na graduação e na pós-graduação, considerando: discentes, educadores, cenários e práticas pedagógicas.

- Apontar estratégias de enfrentamento para lidar com esses desafios ("como lidar"?).

- Elaborar um documento-base para o "I Fórum de Formação em Medicina de Família e Comunidade e Atenção Primária à Saúde", Fortaleza (CE), 01 de maio de 2008.

A partir das idéias levantadas pelas discussões preparatórias para a presente Oficina, realizadas pelo "Grupo de Formadores em MFC e APS”, na lista de discussão virtual da
SBMFC para esse fim, a metodologia utilizada foi a de trabalho em pequenos grupos constituídos pelos diversos atores envolvidos na formação em MFC e APS (docentes, preceptores, residentes, estudantes, profissionais e gestores da saúde). Houve uma dinâmica inicial de integração e apresentação da contextualização e dos objetivos da Oficina. Após, iniciou-se os trabalhos em quatro grupos mistos (divisão aleatória) com a proposta de olhar para o processo de ensino-aprendizagem a fim de definir os "desafios" e "estratégias" na graduação e pós-graduação para a inserção da APS e da MFC. Todo o processo foi facilitado e relatado por voluntários.

\section{Principais resultados*}

Quadro 03. O que lembra "MFC"?

\begin{tabular}{|c|c|}
\hline Esperança (3) & Humanização (2) \\
\hline Desafio (5) & Aprendizagem (2) \\
\hline Sonho & Inovação \\
\hline Aceitação & Cuidado (2) \\
\hline Confiança (2) & Compromisso (2) \\
\hline Integralidade (3) & Cidadania \\
\hline Amor (2) & Dedicação \\
\hline Jeito & Competência \\
\hline Vínculo & Comunicação \\
\hline Longitudinalidade & Suporte \\
\hline Encanto & Curiosidade \\
\hline Integração & União \\
\hline Dom & Alicerce \\
\hline Qualidade & Acolhimento \\
\hline Guerra & Paixão \\
\hline Preconceito & Saúde \\
\hline Complexidade & Adesão \\
\hline Empolgação & Esperança (3) \\
\hline Humanização (2) & Empolgação \\
\hline Desafio (5) & Aprendizagem (2) \\
\hline Sonho & Inovação \\
\hline Aceitação & Cuidado (2) \\
\hline Confiança (2) & Compromisso (2) \\
\hline Integralidade (3) & Cidadania \\
\hline Amor (2) & Dedicação \\
\hline Jeito & Competência \\
\hline Vínculo & Comunicação \\
\hline Longitudinalidade & Suporte \\
\hline Encanto & Curiosidade \\
\hline Integração & União \\
\hline Dom & Alicerce \\
\hline Qualidade & Acolhimento \\
\hline Guerra & Paixão \\
\hline Preconceito & Saúde \\
\hline Complexidade & Adesão \\
\hline
\end{tabular}

*Incluindo também as questões relativas à pós-graduação, haja vista a correlação estratégica com a graduação. 
Quadro 04. Principais desafios da inserção da APS e MFC na Graduação.

- Articulação dos atores

- Valorização da área de conhecimento

- Qualificação dos cenários

- Qualificação e Educação Permanente de profissionais que ensinam e fazem

- Adesão a um novo paradigma de Integralidade das práticas e ensino

- Desarticulação da rede

Quadro 05. Principais desafios da inserção da APS e MFC na Pós-Graduação.

- Superação do desconhecimento e das competências específicas

- Formação Específica do educador técnica e pedagógica

- Organização do processo de trabalho em serviço

- Definição de linhas de pesquisa para produção de conhecimento

- Cadeia graduação-pós-serviço

- Gestão Compartilhada da cadeia de formação

Quadro 06. Principais estratégias para superar os desafios na Graduação.

- Articulação Academia Serviço Comunidade Gestor planejamento e avaliação conjuntos.

- Definir claramente os papéis de cada um.

- Utilizar mecanismos de indução disponíveis hoje.

- Fóruns regulares de divulgação dos princípios da APS E MFC - a divulgação está em nós mesmos.

- Geração de conhecimentos que qualifiquem e demonstrem a pertinência destas estratégias.

- Indicadores de qualidade para os cenários de prática.

- Co-responsabilizar a academia pelo processo de educação permanente do serviço.

- Fazer pactos definidos de trabalho.

- A graduação tem de utilizar a pós-graduação para melhorar o cenário de práticas.

- Estabelecer diretrizes de articulação do ensino com os serviços (da academia com a rede de gestão), pactuadas por meio de comissões, com a participação dos diversos atores do SUS e IES.

- Formalizar a articulação Ensino - Serviços através de convênios ou contratos entre as partes, aprovado pelos órgãos de controle social correspondente (Conselhos de unidades, distritais, municipais e estaduais).

- Definir portaria do SUS sobre as diretrizes de artivculação Ensino - Serviço, estabelecendo prioridade para as IES comprometidas com a formação de profissionais com perfil adequado a demanda do SUS.

- Política de educação permanente.

- Estímulo à pesquisa sobre a prática e a gestão.

- Formar pessoas com a visão do novo paradigma - diretrizes curriculares.

- Política Geral de valorização da APS e MFC. 
Quadro 07. Principais estratégias para superar os desafios na pós-graduação.

- Desenvolver oficinas junto aos gestores de sensibilização para a oportunidade que a APS e a MFC têm de satisfazer a população e economizar recursos.

- Definir currículo por competências.

- Expandir em quantidade e qualidade as oficinas de formação de preceptores efacilitadores.

- Incorporação do Médico de Família e Comunidade nos aparelhos formadores.

- Capacitação em pesquisa na APS.

- Formação pedagógica - metodologias ativas e multiprofissionais.

- Academicizar o serviço e serviçalizar a academia.

- Identificar necessidades e compartilhar resultados de forma integrada.

- Curto/Médio prazo - incentivar os programas que existem para que eles identifiquem linhas de pesquisa de APS e MFC.

- Médio/Curto prazo - criar produtos novos de formação mestrado e doutorado com respectivas linhas de pesquisa.

- Olhar local/regional e nacional.

- Construir parcerias interinstitucionais para construir observatórios, núcleos e linhas de pesquisa.

- Pressionar agências de fomento para editais de pesquisa na área.

- Contribuir e realizar os processos de avaliação das mudanças curriculares redirecionando a pesquisa.

- Envolvimento nos planos locais e regionais para indicar cenários de práticas possíveis.

- Aparelhar instituições acadêmicas para interferir na pactuação.

- Oferecer capacitação aos gestores - pessoas envolvidas nos projetos de construção de projetos e intervenção, ainda que com gestão informal.

- Pós-graduação com competências gerenciais e linhas de pesquisa que apontem para a discussão política.

- Plano regional de desenvolvimento de educação e serviço - antigas rodas que necessitam serem "ocupadas" na lógica sistêmica, e não disciplinar exclusiva.

\section{Considerações finais.}

A inserção dos princípios e das práticas da MFC dentro do currículo de graduação em Medicina representa uma estratégia fundamental para a formação de futuros médicos que tenham o cuidado como eixo central de sua prática e que desenvolvam o senso de co-responsabilidade pela saúde integral das pessoas. Além de tudo, que sejam engajados e comprometidos com o Sistema Único de Saúde, conforme as Diretrizes Curriculares Nacionais do Curso de Graduação em Medicina.

Dentre os principais entraves atuais para que a inserção da MFC no currículo das escolas médicas aconteça de forma mais intensa, pode-se citar a não-existência de uma política nacional com esta finalidade, apesar de já existirem várias experiências nesse sentido e das diretrizes internacionais no tema.

O presente documento procura trazer informações para que as Escolas Médicas se instrumentalizem e operacionalizem a inserção da APS e da MFC nos cursos de graduação.

Algumas questões necessitariam de um maior delineamento:

Considerando que a MFC deve ter um caráter transversal na formação do estudante, qual o período mais adequado para iniciar a inserção no currículo (mais precoce ou mais tardia)? Como promover a relação/integração com as outras disciplinas da faculdade/curso de Medicina? Como equilibrar e guiar a relação das instituições de ensino e o sistema de saúde público (integração ensinoserviço-comunidade), em especial em relação à esfera municipal, relação esta necessária e fundamental para o sucesso 
da inserção, segundo as próprias Diretrizes Curriculares Nacionais do Curso de Graduação em Medicina do MEC?

Entende-se que a SBMFC, enquanto sociedade cientifica da especialidade, em esforço conjunto com a ABEM, MEC e MS, tem papel estratégico no sentido de sensibilizar as escolas médicas, apoiar e assessorar a criação de estruturas acadêmicas e docente-assistenciais na especialidade, bem como elaborar diretrizes curriculares específicas para a inserção da MFC na graduação em Medicina.

A Medicina de Família e Comunidade: tem potencial para colaborar na transformação de um sistema de saúde nacional, ainda fragmentado, em outro integral e integrado, com abordagem generalista e humana, por meio de uma prática multi e interdisciplinar, eliminando barreiras ao acesso, com sistema de informação avançado e informatizado, e com foco na qualidade da assistência e na racionalização dos recursos financeiros. Apesar do enorme desafio e das grandes dificuldades, a SBMFC se coloca à disposição para enfrentar o processo, entendendo ser o ensino de graduação uma estratégia fundamental, e, por isso, o porquê deste documento.

\section{Referências.}

1. Justin Allen et al. Definição Européia de Clínica Geral e Medicina Familiar. [s.l.]: Wonca - Europa; 2002.

2. Byrne PS, et al. The Contribution of the General Practitioner to Undergraduate Medical Education. A Statement by the working party appointed by the second European Conference on the Teaching of General Practice (Leeuwenhorst Netherlands, 1974). Netherlands: [s.n.]; 1977.

3. Relatório da Conferência conjunta da OMS e da WONCA em Ontário - Canadá. Haciendo el ejercicio médico y la formación médica más adecuados a las necesidades de la población: la contribución del médico de família. [s.l.]: Sociedad Española de Medicina de Familia y Comunitária; 1994.

4. Rodgers et al. Family Medicine. 2002; 34 (4):237-9.

5. Bucholtz JR et al. The FMCR Project's FM Clerkship/ Post Clerkship Workgroup. Future of Family Medicine (FFM) Project. Annals of Family Medicine. Mar. 2004; (suppl.).

6. Associação Paulista de Medicina de Família e Comunidade. I Congresso Paulista de Medicina de Família e Comunidade. Revista Medicina. Set.; 2005; (supl.7).

7. Gomes LFRA, Demarzo MM. P. Tradução da Agenda Educativa da Academia Européia de Medicina de Família e Comunidade. Rev Bras Med Família e Comunidade. 2006; 5 (supl.): 77-124.

8. Brasil. Ministério da Educação. Diretrizes curriculares nacionais do curso de graduação em medicina. Conselho Nacional de Educação - Câmara de Educação Superior Resolução CNE/CES no 4, de 7 de Novembro de 2001. 9. Brasil. Ministério da Saúde. Ministério da Educação. A aderência dos cursos de graduação em enfermagem, medicina e odontologia às Diretrizes Curriculares Nacionais. Brasília (DF): Ministério da Saúde; 2006.

10. Associação Paulista de Medicina de Família e Comunidade. I Mostra de medicina de família e comunidade na graduação. I Congresso Paulista de Medicina de Família e Comunidade. Rev Medicina. Set. 2005; (supl.7).

11. Organização Mundial de Saúde. Organização Mundial dos Médicos de Família. Improving Health Systems: the contribution of family medicine. [s.l.]: Wonca; 2002.

12. Demarzo MMP, Anderson MIP. Apresentação da II Mostra de Medicina de Família e Comunidade na Graduação II Mostra Nacional de Medicina de Família e Comunidade na Graduação, 2006, Gramado-RS. Rev Bras Med Família e Comunidade. 2006; 5 (supl): 6-10.

13. Oliveira AMF, Brunelli B, Kanno NP, Lora PST. Apresentação do I Encontro de Ligas Acadêmicas de Medicina de Família e Comunidade. In: II Mostra Nacional de Medicina de Família e Comunidade na Graduação, 2006, Gramado-RS. Revista Brasileira de Medicina de Família e Comunidade. Rev Bras Med Família e Comunidade. 2006; 5 (supl): 11-15.

14. Sociedade Brasileira de Medicina de Família e Comunidade. Desafios do Ensino e da Aprendizagem da Atenção Primária à Saúde e da Medicina de Família e Comunidade na Graduação e Pós-Graduação em Medicina. Relatório de Oficina. $45^{\circ}$ Congresso Brasileiro de Educação 
Médica (COBEM), Associação Brasileira de Educação

Médica (ABEM), Uberlândia-MG, 20 de outubro de 2007.

Disponível em: www.sbmfc.org.br.

Documento Oficial da Sociedade Brasileira de Medicina de Família e Comunidade (SBMFC)

\section{Endereço para Correspondência:}

Rua 28 de setembro, sala 605,

Rio de Janeiro RJ

CEP: 20.551-031

\section{Endereço Eletrônico:}

inezpadula@terra.com.br 